\title{
Impact of Mesh Quality Improvement Systems on the Accuracy of Adaptive Finite-Element Electromagnetics With Tetrahedra
}

\author{
Mark Dorica and Dennis D. Giannacopoulos, Member, IEEE \\ Department of Electrical and Computer Engineering, McGill University, Montreal, QC H3A 2A7 Canada
}

Finite-element (FE) accuracy can be directly affected by mesh quality. The potential benefits and related costs of a family of new mesh quality improvement systems are investigated using a suite of electromagnetic benchmarks and mesh quality measures theoretically linked to FE accuracy. Experimental findings suggest error reductions on the order of $10 \%$ for half-second runtimes.

Index Terms-Adaptive systems, electromagnetic analysis, finite-element methods (FEMs), mesh improvement, mesh quality.

\section{INTRODUCTION}

$\mathbf{I}$ $\mathrm{T}$ is well known that the accuracy and efficiency of the finite-element method (FEM) can be negatively affected by very few poor quality tetrahedral elements [1]-[4]. In certain cases, a single bad element can cause a high-discretization error throughout the entire problem domain. When employing $h$-adaptive FEMs for electromagnetics, a mesh improvement stage is required in order to ensure high-quality tetrahedra [5]-[7]. However, mesh improvement techniques and quality indicators are often subjective or based on purely geometrical considerations [4]. Several decades after the invention of the FEM, the connections between element shape and accuracy are yet to be solidified.

Recent work in finite-element (FE) approximation theory has focused on theoretically linking mesh quality to interpolation and discretization errors [1]-[4]. The resulting error bounds and first-order tetrahedral element quality indicators may be used to directly evaluate the performance of mesh improvement systems in terms of FE accuracy. Based on these indicators, one may also formulate new mesh improvement systems that effectively target the most problematic elements.

Novel mesh improvement techniques D1-D4 were shown to outperform previous combined mesh smoothing techniques in geometric mesh quality [5]. This work focuses on evaluating the performance of the new family of mesh smoothing systems using the proposed theoretical mesh quality indicators. The potential benefits and related costs of the new techniques for $h$-adaptive FE electromagnetic analysis will be examined. Experimental findings will highlight their practical advantages and corroborate the theoretical results.

\section{Mesh Quality Challenge in AdAPTIVE FEMS}

The number and distribution of degrees of freedom (DOF), as well as the shape of the elements has a direct influence on the resulting accuracy of the FE solution. For $h$-adaptive FEMs with first-order tetrahedral elements, the first two criteria are tied to

Digital Object Identifier 10.1109/TMAG.2005.846079

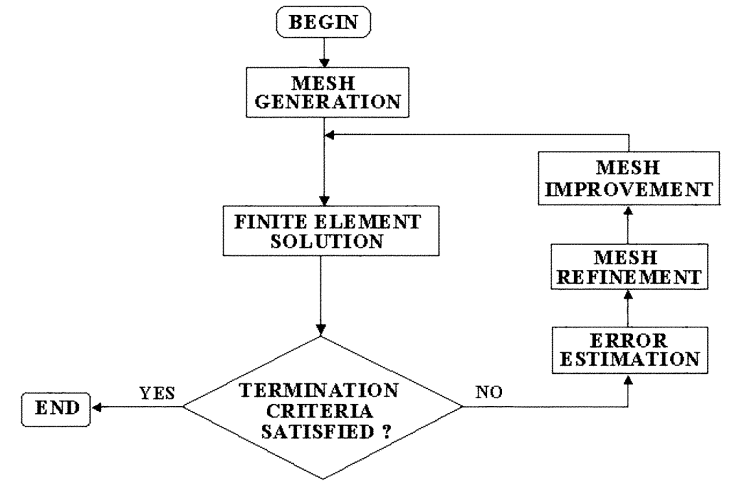

Fig. 1. $\quad h$-adaptive FE analysis model.

the manner in which elements are added to the discretization during the refinement stage. In essence, adaptive FEMs strive to achieve a specific level of accuracy for the minimum computational cost by intelligently evolving the mesh. The number and distribution of tetrahedra is initially established during the mesh generation phase and is repeatedly refined based on an error map. As can be observed in Fig. 1, the first two accuracy-related considerations (noted above) are integral parts of the overall adaptive paradigm.

Close attention must be paid to element shape during each iteration of the adaptive process. While certain refinement algorithms such as edge bisection and Delaunay refinement have shown promise, a large percentage of the added elements can still exhibit poor quality [3]-[6]. These elements can significantly degrade the accuracy of the solution. For this reason, the development of an efficient mesh improvement stage is considered a priority for $h$-adaptive FE electromagnetic analysis [5]-[7]. Prior to selecting a mesh improvement algorithm, a theoretical basis must be established for detecting poor quality elements and making fine distinctions between mesh improvement systems.

\section{THEORETICAL MESH QUALITY INDICATORS}

From engineering experience, tetrahedra diverging from the equilateral shape are usually considered to be "poor." A sampling of such tetrahedra is shown in Fig. 2. This purely geo- 


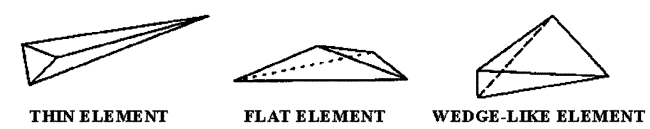

Fig. 2. Sampling of tetrahedra generally considered to be poor.

TABLE I

SUITE OF MESH QUALITY INDICATORS

\begin{tabular}{c|c|c|c|c}
\hline INDICATOR: & M1 & M2 & M3 & M4 \\
\hline FORMULA: & $6 \sqrt{2} \frac{V}{l_{\text {rms }}^{3}}$ & $V\left[\frac{\sum_{m=1}^{4} A_{m}}{\sum_{1 \leqslant i j \leq s} A_{i} A_{j} l_{i j}^{2}}\right]$ & $\mathbf{2} \sqrt{6}\left(\frac{R_{i}}{l_{\max }}\right)$ & $\sigma_{\min }(E)$ \\
\hline
\end{tabular}

metric interpretation is useful as a rule of thumb, but fails to fully characterize the links between mesh quality and FE accuracy. Recent theoretical advances have shed light on the topic [1]-[4].

Table I lists a selection of mesh quality indicators for firstorder tetrahedral elements. In this table, $V$ is the tetrahedral volume, $l_{\mathrm{rms}}$ is the rms edge length of the element, $A_{i}$ is the area of a triangular face, $i, l_{i j}$ is the length of the edge connecting vertices, $i$ and $j, R_{i}$ are the radius of the inscribed sphere, $l_{\max }$ is the maximum edge length, $\sigma_{\min }(\cdot)$ is the minimum singular value operator, and $E$ is the edge shape matrix. The $E$ matrix is $3 \times 6$ and has unit vector columns directed along the element edges (in either of the two possible directions).

The M1 measure is a successful purely geometric measure that serves to bridge the gap with previous efforts in geometric mesh quality analysis [8]. Conversely, M2, M3, and M4 are all theoretically linked to FE approximation accuracy. All measures are scale invariant so that only the impact of shape (not size) is evaluated.

The M2 measure is based on a first-order tetrahedron's interpolation fitness [4]. There is a close link between discretization and interpolation errors. When investigating the interpolation fitness of first-order tetrahedral FEs, interpolation error is discussed in two veins: 1) the difference between the interpolated function and the exact solution and 2) the difference between the gradient of the interpolated function and the gradient of the exact solution.

Strategies for reducing interpolation error often have a similar effect on discretization error. However, the relationship between discretization error and tetrahedral mesh quality cannot be fully characterized without knowledge of the partial differential equation being solved [4]. The maximum pointwise elemental interpolation error bounds can be reduced by using smaller elements. Hence, the shape of elements is usually controlled by the need to bound the maximum elemental pointwise error in the interpolated gradient, as it is much more sensitive to poor tetrahedral shapes (i.e., growing without bound for angles approaching $180^{\circ}$ [4]). The $\mathrm{M} 2$ measure is based on this latter consideration. In general, bounds on FE discretization error can only be obtained if both interpolation errors are bounded.

The M3 measure has a solid theoretical basis linking it to approximation accuracy for first-order tetrahedral FEs [4]. In particular, M3 is linked to interpolation error bounds suggested by approximation theory.

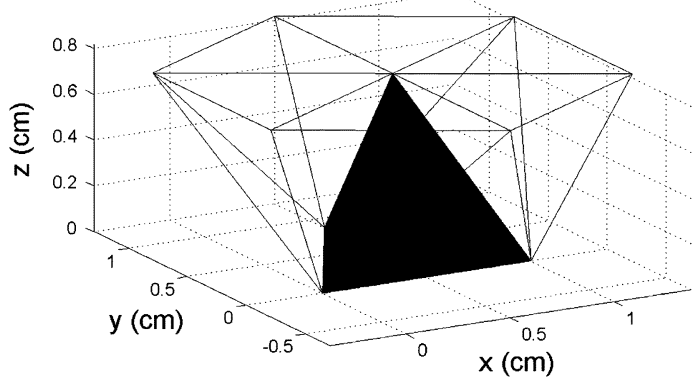

Fig. 3. Mesh for lower half of 3-D electrostatic test case.

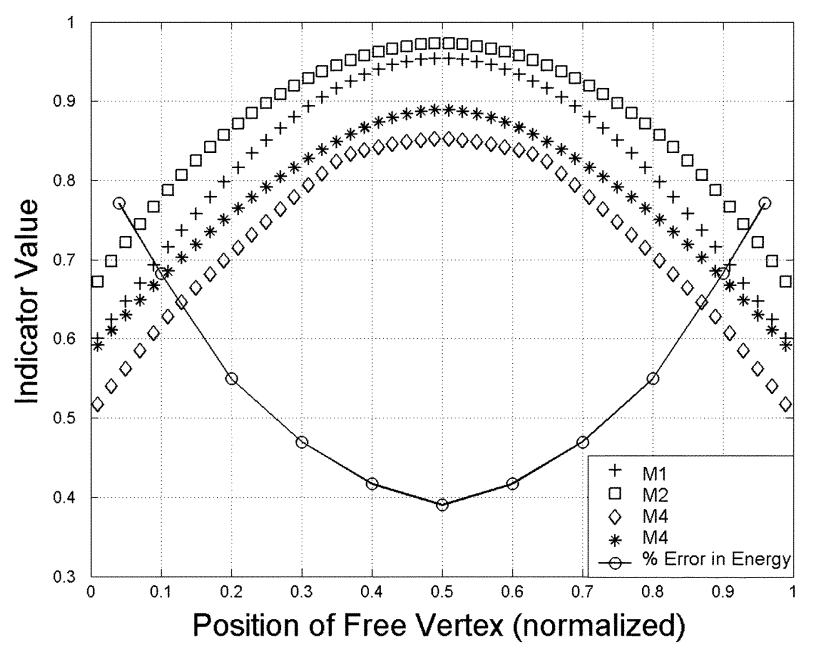

Fig. 4. Mean indicator values identify optimal position of interior vertex yielding a $40 \%$ reduction in energy error.

The M4 measure is Tsukerman's minimum singular value condition for tetrahedra [1]-[3]. Tsukerman characterizes the shape of a tetrahedron by the edge shape matrix $E$ (described earlier). The minimum singular value of $E$ is linked to the proximity of the unit edge vectors (columns) to one plane. M4 is the governing factor for FE interpolation errors in first-order tetrahedral approximations of conservative fields [1].

The M1-M4 mesh quality indicators can be validated through a basic three-dimensional (3-D) electrostatic Laplace test. The system consists of a base "bowl" joined to a top "cap" (mirror image of Fig. 3) across a shared hexagonal plane [9]. All exterior cap faces are set to $1 \mathrm{~V}$; the triangular base of the bowl is set to $0 \mathrm{~V}$. A hexagonal plane position midway between the base bowl and top cap yields the results in Fig. 4. Movement of the interior (free) vertex toward either extreme creates flat, elongated, and distorted tetrahedra (similar to those in Fig. 2). M1-M4 correctly identify the optimal position for the interior vertex, yielding a $40 \%$ reduction in energy error as compared to the extreme cases (each close to $80 \%$ error). Numerous test cases based on this geometry were used to validate M1-M4.

\section{Mesh Quality ImProvement Systems}

The impact of mesh quality on accuracy is of increasing importance as the range and complexity of FE applications is continually expanding. When large numbers of DOF are required to achieve the highest levels of accuracy, it is desirable to avoid 
unnecessary adaptive passes. For a relatively small cost, a mesh quality improvement stage can provide a marked increase in accuracy while maintaining a fixed number of DOF. Occasionally, poor mesh quality can counteract increases in DOF. This leads to unsatisfactory accuracy at even the highest levels of refinement. Therefore, considerable attention has been given to mesh quality improvement over the past several years. Among the most promising mesh quality improvement methods for 3-D meshes are smoothing-based techniques [5]-[7], [10].

Mesh smoothing techniques reposition individual vertices within the mesh to improve local mesh quality without changing the mesh connectivity. Typically, several iterations of smoothing are performed to improve the overall quality of the mesh. Smart-Laplacian smoothing and optimization-based smoothing are two variants that have received much attention [5], [6], [10]. Smart-Laplacian smoothing repositions a vertex to the average location of the vertices connected to it by edges only if the quality of the local submesh is improved according to a specific quality measure. This yields an improved, yet nonoptimal local submesh. Optimization-based approaches formulate the smoothing operation as a nonsmooth optimization problem, and use an analogue of the steepest descent method for smooth functions to maximize or minimize a given mesh quality measure [10]. This yields an optimal local submesh. However, computational experiments have shown that optimization-based approaches can be up to 10 times more expensive than smart-Laplacian smoothing [10]. To circumvent this problem, novel mesh smoothing techniques D1-D4 based on combinations of smart-Laplacian smoothing and optimization-based smoothing were presented in [5]. The new techniques D1-D4 were able to outperform or match the existing techniques in both computational cost and mesh quality improvement (as measured by dihedral angle distributions). Using a combined theoretical and experimental approach, the relation to FE accuracy will now be addressed.

\section{RESUlts}

The suite of theoretical indicators of Table I will now be used to demonstrate the accuracy enhancement offered by the new mesh smoothing techniques. An analogous approach was used for determining the best mesh refinement strategy in [3]. The error in electrostatic energy will highlight practical advantages of the smoothing systems.

The 3-D electrostatic benchmark in Fig. 5 consists of one-eighth of an air-filled, concentric, cuboidal capacitor [9]. This benchmark incorporates common problematic features of practical structures, including sharp edges and corners. These features make it useful for drawing comparisons between the smoothing techniques. The conductor boundary conditions are $1 \mathrm{~V}$ on the small, inner cube and $0 \mathrm{~V}$ on the outer cube.

An $h$-adaptive system of the type depicted in Fig. 1 is simulated in order to assess the impact of the new mesh improvement systems on the accuracy of the electrostatic solution. Hence, test case \#1 simulates a first adaptive pass where the initial mesh (633 tets) is $h$-refined by adaptively focusing elements near the inner conducting cube (1061 tets) and, subsequently, improved using a given mesh improvement system. Test case

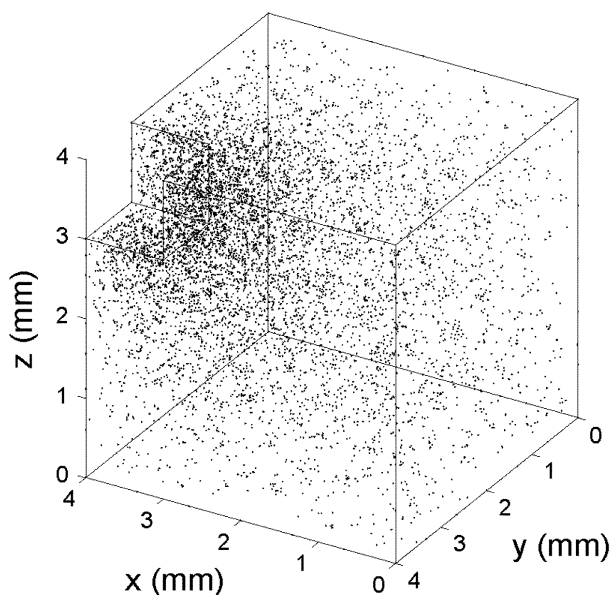

Fig. 5. Cuboidal capacitor 3-D test problem (one-eighth geometry) and vertex distribution for adaptively refined mesh (test case \#2).

TABLE II

Evaluation of SMoOthing TeChNiQues For TEST CASE \#2.

\begin{tabular}{c|c|c|c|c|c|c|c|c|c|c|c}
\hline \multirow{2}{*}{ INDIC } & \multirow{2}{*}{ REFINED } & \multicolumn{10}{|c|}{ IMPROVED } \\
\cline { 3 - 13 } & C1 & C2 & C3 & C4 & C1f & C3f & D1 & D2 & D3 & D4 \\
\hline M1 & 0.7360 & 0.7402 & 0.7479 & 0.7257 & 0.7497 & 0.7433 & 0.7272 & 0.7689 & 0.7598 & 0.7686 & 0.7707 \\
\hline M2 & 0.7913 & 0.8005 & 0.8054 & 0.7894 & 0.8066 & 0.8023 & 0.7907 & 0.8201 & 0.8127 & 0.8195 & 0.8207 \\
\hline M3 & 0.6392 & 0.6450 & 0.6520 & 0.6299 & 0.6536 & 0.6476 & 0.6312 & 0.6717 & 0.6610 & 0.6714 & 0.6736 \\
\hline M4 & 0.6735 & 0.6810 & 0.6859 & 0.6703 & 0.6870 & 0.6827 & 0.6712 & 0.6996 & 0.6929 & 0.6993 & 0.7009 \\
\hline
\end{tabular}

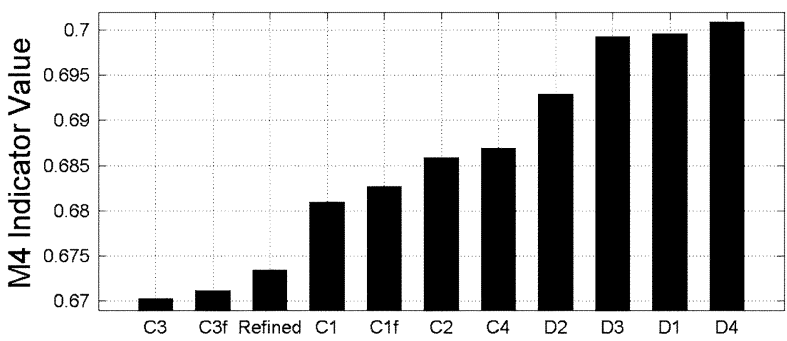

Fig. 6. Mean values of the M4 accuracy indicator for test case \#2. All indicators produce this same ranking for both test cases.

\#2 uses an analogous approach for simulating the final adaptive pass. The mesh sizes for test case \#2 are considerably larger: 20409 and 25 483, respectively. The test case \#2 vertex distribution is shown in Fig. 5.

Table II presents test case \#2 values for mesh quality before ("refined") and after ("improved") smoothing. The indicators have been normalized such that their minimal value is zero (degenerate tetrahedron) and maximal value is one (highest quality). Recall that M1-M4 cover the spectrum of theoretical solution accuracy and geometric mesh quality. The mean values of M1-M4 in Table II are fully representative of test case \#1 performance as well. In particular, both sets of results yield the same performance ranking of the smoothing systems. This ranking is evident in Fig. 6, where D4, D1, D3, and D2 are clearly superior for the M4 indicator. As can be readily observed in Table II, M1-M3 are in agreement with the ranking in Fig. 6. To summarize, D1-D4 outperform C1-C3f [10] for the entire suite of indicators and both test cases. Furthermore, M1-M4 all classify the new techniques in the same order of effectiveness: D4, D1, D3, and D2 for both test cases. 


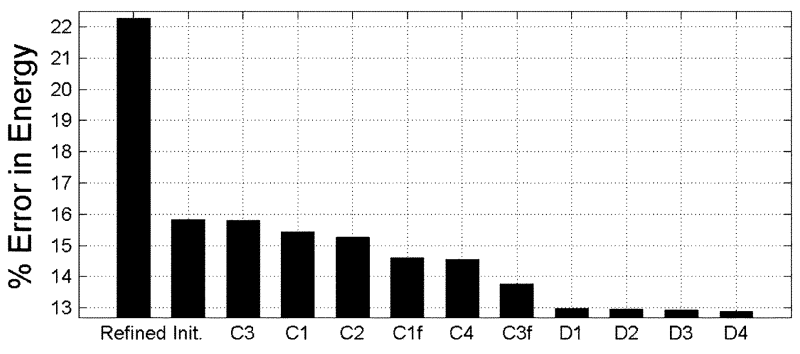

Fig. 7. Energy error reduction through mesh improvement for test case \#1.

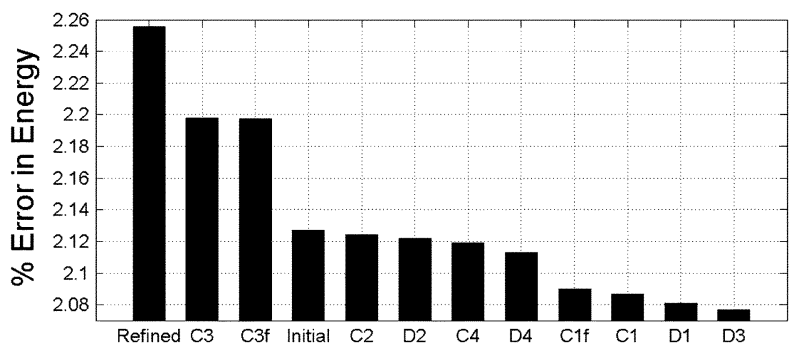

Fig. 8. Energy error reduction through mesh improvement for test case \#2.

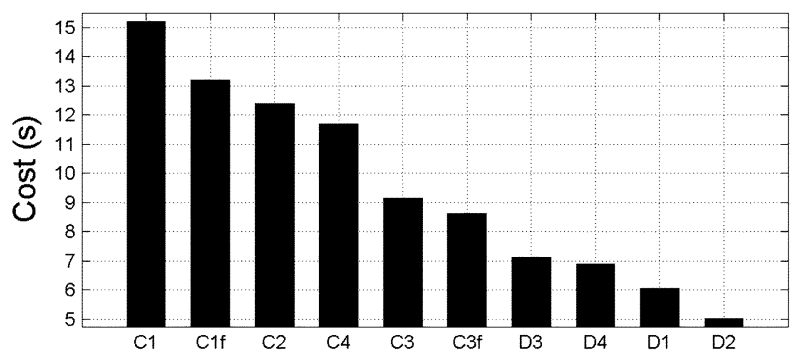

Fig. 9. Smoothing times for test case \#2. Test case \#1 provides an analogous ranking with timings near the 0.5-s mark.

Errors in electrostatic energy are now used to determine the practical implications of these smoothing systems. In Fig. 7, notice that refinement of the test case \#1 initial mesh increased the energy error by $6 \%$. This is an example of how a refined yet poor quality 1061 -element mesh (22\% error) can be less accurate than an initial 633-element mesh (16\% error). D1-D4 all provide a $10 \%$ reduction in error relative to the refined mesh. Remarkably, these test case \#1 results were obtained for smoothing times on the order of one-half second.

In Fig. 8, it can be observed that test case \#2 is on a higher accuracy plateau. D3 and D1 produce the best results and reduce the error by as much as $0.18 \%$. While this reduction in error is smaller than in test case \#1, it is quite significant. The smoothing systems maximize the benefit of each DOF and provide a more reliable mesh for further adaptive passes.

The smoothing times in Fig. 9 corroborate earlier findings in [5]. Specifically, D1-D4 succeed in substantially reducing practical costs for test case \#2 mesh improvement. While the test case \#1 results produce the same ranking, the timings are an order of magnitude smaller (half-second range).

The experimental results and M1-M4 indicators are meant to complement each other rather than to provide identical matches.
Mean theoretical indicator values based on error bounds cannot replace an experimental evaluation of effectiveness. For example, minimizing the maximum bound on interpolation error is not the same as minimizing the maximum interpolation error. Furthermore, a poor quality element located in a critical part of the mesh has a greater impact than one located in a less critical location. Also, poor elements have a greater relative effect in small meshes. A mean indicator value may not capture all these fine nuances. Customizing the M1-M4 indicators based on the partial differential equation would yield closer matches between theory and experiment, but would lead to a loss of generality.

\section{CONCLUSION}

The impact of mesh quality on FE accuracy should not be underestimated in today's complex modeling environments. Powerful and efficient mesh improvement systems are useful tools for achieving substantial increases in accuracy and reducing the number of adaptive passes. Novel smoothing-based mesh improvement techniques D1, D2, D3, and D4 for $h$-adaptive FE analysis with tetrahedra described in [5] were compared with existing techniques using a suite of state-of-the-art theoretical accuracy indicators and standard electromagnetic benchmark tests. The new techniques D1-D4 were able to outperform the existing techniques in FE accuracy enhancement, mesh quality improvement, and computational cost.

\section{ACKNOWLEDGMENT}

This work was supported in part by the Natural Sciences and Engineering Research Council of Canada.

\section{REFERENCES}

[1] I. Tsukerman, "A general accuracy criterion for finite element approximation,” IEEE Trans. Magn., vol. 34, no. 5, pp. 2425-2428, Sep. 1998.

[2] — "Approximation of conservative fields and the element edge shape matrix," IEEE Trans. Magn., vol. 34, no. 5, pp. 3248-3251, Sep. 1998.

[3] - "Refinement strategies and approximation errors for tetrahedral elements," IEEE Trans. Magn., vol. 35, no. 3, pp. 1342-1345, May 1999.

[4] J. R. Shewchuk, "What is a good linear element," in Proc. 11th Int. Meshing Roundtable, Albuquerque, NM, Sep. 2002, pp. 115-126.

[5] M. Dorica and D. Giannacopoulos, "Toward optimal mesh quality improvements for adaptive finite element electromagnetics with tetrahedra," IEEE Trans. Magn., vol. 40, no. 2, pp. 989-992, Mar. 2004.

[6] J.-F. Lee, R. Dyczij-Edlinger, E. Lucas, and T. Fontana, “Application of steepest descent smoothing and de-meshing techniques in $h$-version adaptive FEMs,' IEEE Trans. Magn., vol. 35, no. 3, pp. 1346-1349, May 1999.

[7] D. Dyck, D. A. Lowther, W. Mai, and G. Henneberger, "Three-dimensional mesh improvement using self organizing feature maps," IEEE Trans. Magn., vol. 35, no. 3, pp. 1334-1337, May 1999.

[8] V. N. Parthasarathy, "A comparison of tetrahedron quality measures," Finite Elements Anal. Design, vol. 15, pp. 255-261, 1993.

[9] D. Giannacopoulos and S. McFee, "Functional derivatives and optimal discretization based refinement criteria for adaptive finite element analysis with scalar tetrahedra," IEEE Trans. Magn., vol. 35, no. 3, pp. 1326-1329, May 1999.

[10] L. A. Freitag and C. Ollivier-Gooch, "Tetrahedral mesh improvement using swapping and smoothing," Int. J. Numer. Meth. Eng., vol. 40, pp. 3979-4002, 1997.

Manuscript received June 8, 2004. 